\title{
Study on the Retarding Mechanism and Strength Loss of Gypsum from Hydrolyzed Wheat Protein Retarder
}

\author{
Yi Ding****, Youchun Fang****, Hui Fang****, Qicai Zhang****, Fengjun Zhang****, \\ and Won-Chun Oh****t+ \\ *Anhui Key Laboratory of Advanced Building Materials, Anhui Jianzhu University, Hefei Anhui 230022, P R China \\ **Key Laboratory of Functional Molecule Design and Interface Process, Anhui Jianzhu University, Hefei Anhui 230601, P R China \\ ***Department of Advanced Materials Science \& Engineering, Hanseo University, Seosan 356-706, Korea
}

(Received October 20, 2014; Accepted December 1, 2014)

\begin{abstract}
In this article, the influence of a hydrolyzed wheat protein retarder on the hydration process, ion concentration in liquid phase, degree of supersaturation, and crystal morphology of plaster was investigated. Furthermore, the retarding mechanism and the strength loss of gypsum were also studied by scanning electron microscopy (SEM). The results indicate that the use of the hydrolyzed wheat protein retarder for plaster achieved a better retarding effect and lower strength loss. The combination of gypsum plaster with the retarder not only decreased the plaster's early hydration rate and prolonged its setting time efficiently, but also militated against the crystal morphology of dihydrate gypsum. For example, the crystal dimensions changed little, but the proportion of needle-shaped crystals decreased. Combination with calcium ions on the surface of dihydrate gypsum crystal nuclei may form a chemisorbed layer, reduce the surface energy of the crystal nuclei, and inhibit the growth of the crystal nuclei of dihydrate gypsum. Consequently, the hydration process of building gypsum becomes greatly extended and is slowed down significantly.
\end{abstract}

Key words : FGD gypsum, Retarder, Hydration process, Crystal microstructure, Retarding mechanism

\section{Introduction}

$\mathrm{T}$ raditional annexing agents, such as sodium hexametaphosphate, citric acid, and its salt, are commonly used in high-efficiency gypsum retarders, but they have a negative impact on the strength of gypsum. A protein macromolecule retarder has the advantages of lower accretion and better retarding effect, and it results in less strength loss. ${ }^{1)}$

Retarders with different retarding mechanisms have varying effects on the strength of gypsum. In this regard, hydrolyzed wheat protein retarder has less negative effect. Many scholars throughout the world ${ }^{2-4)}$ have conducted extensive research on the effects of gypsum retarders, the hydration process and retarding mechanisms. Previous studies of gypsum retarder have mainly focused on small molecule retarders but not macromolecular retarders. ${ }^{5-8)}$ Also, there have been very few studies on the effects of macromolecular retarders on the hydration process of building gypsum, the microstructure of the hardened body, the inner causes of strength loss, and retardation mechanisms. Therefore, in this article, we investigated the influence of a mac-

\footnotetext{
${ }^{\dagger}$ Corresponding author : Yi Ding

E-mail : dyrqf@aiai.edu.cn

Tel : +86-551-6382-8262 Fax : +86-551-6382-8106

${ }^{\dagger}$ Corresponding author: Won-Chun Oh

E-mail : wc_oh@hanseo.ac.kr

Tel : +82-41-660-1337 Fax : +82-41-688-3352
}

romolecular retarder on the setting time of desulfurized gypsum, the hydration process and crystal morphology of dihydrate gypsum, and the retarding mechanism of the macromolecular retarder.

\section{Experimental Procedure}

\subsection{Raw materials}

FGD gypsum was obtained from a Huainan heat-engine plant with the content of $71.4 \%$ dihydrate gypsum and $14.7 \%$ semi-hydrated gypsum, of which the specific surface area was $0.267 \mathrm{~m}^{2} / \mathrm{g}$. The TG analysis values are shown in Fig. 1. The water-gypsum ratio of normal consistency is 0.59 . The hydrolyzed wheat protein retarder, which is called QWP-40, is a commercial industrial product.

\subsection{Test methods}

GB 9776-88 building gypsum was used in the tests to measure its setting time and strength. The crystal morphology of the middle original section of the hardened body was analyzed by SEM. The $\mathrm{Ca}^{2+}$ concentration was determined by atomic absorption spectrometry (AAS), and the $\mathrm{SO}_{4}{ }^{2-}$ concentration was determined by the weighing method of $\mathrm{BaSO}_{4}$.

\section{Results and Discussion}

\subsection{The impact on the hydration process}

As shown in Fig. 2, the setting time of FGD gypsum was 


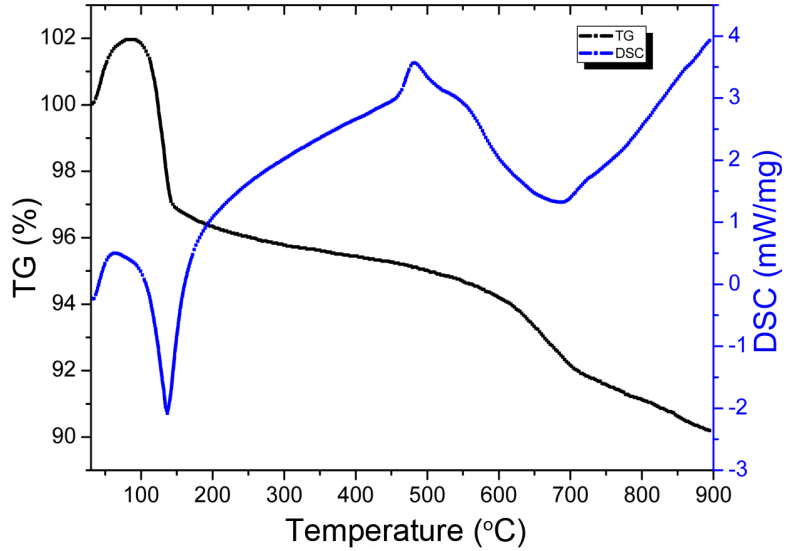

Fig. 1. TG analysis curve of $\beta$ - semi-hydrated gypsum.

gradually extended with increase amounts of the hydrolyzed wheat protein retarder. The initial setting time and final setting time respectively increased to 8 and $16.5 \mathrm{~min}$ with a $0.1 \%$ proportion of the retarder and increased to 17 and 25.5 min with $0.25 \%$. The difference in the setting time also changed. For example, it changed little with the retarder proportion of $0.05 \%$, but it was extended by $3 \sim 4$ min with $0.1 \%$. The trend of setting time maintained steady growth with the increase of retarder. Thus, it would be conducive to operational control, and we could arbitrarily control the setting time according to the operation time.

The flexural and compressive strength also changed. As shown in Fig. 3, the flexural and compressive strength of the desulfurized gypsum slightly decreased when the hydrolyzed wheat protein retarder added. With an increased amount of retarder, the flexural and compressive strength of FGD gypsum showed little fluctuation. This indicates that QWP-40 led to less strength loss of plaster than other retarders, and this could make it an ideal building gypsum retarder.

As shown in Fig. 4, the hydration exothermic process of building gypsum can be divided into three stages. Gypsum releases the heat of solution in the first stage when it comes into contact with water; the hydration heat began to increase slowly in this early stage. The second stage is the period of acceleration, in which the release of hydrationheat appears as a peak, and the total hydration heat rises rapidly. The release of hydration-heat slows down, and the total hydration heat tends towards stability gradually in the third stage. Seen from the curve of the control sample, the accelerating stage of the hydration-heat release corresponded to the time period from the initial setting to the final setting time, and the total hydration heat quantity tended to be stable after the final setting time. After the retarder in the proportion of $0.2 \%$, the early rate of hydration-heat release significantly decreased, and the time that the peak of hydration-heat release appeared was obviously later than that of the control sample. This indicates that the induction period before the initial setting was the prepara-

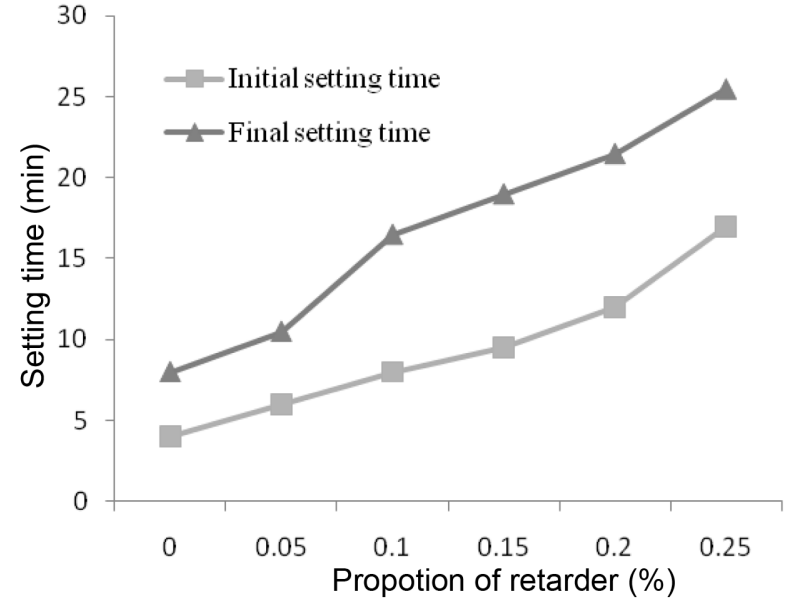

Fig. 2. The influences of retarder proportion on the setting time of FGD gypsum.

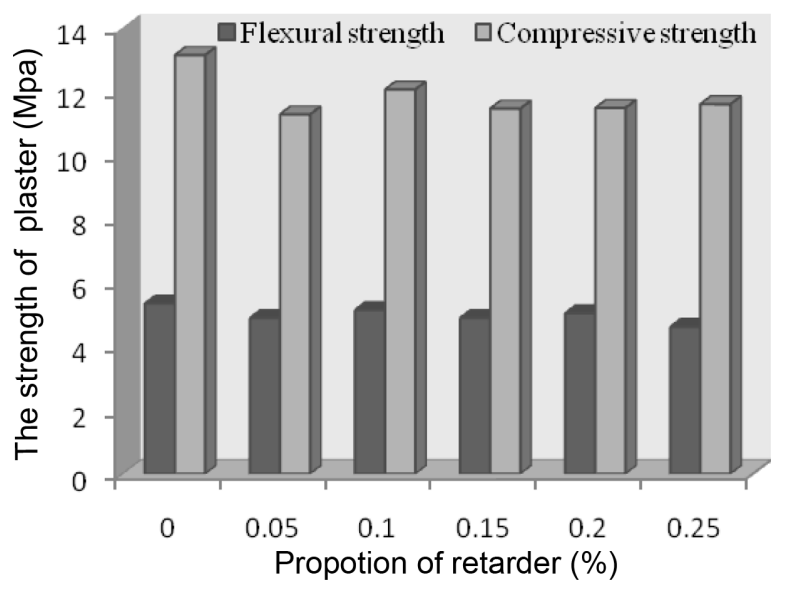

Fig. 3. Influences of retarder proportion on the strength of FGD gypsum.

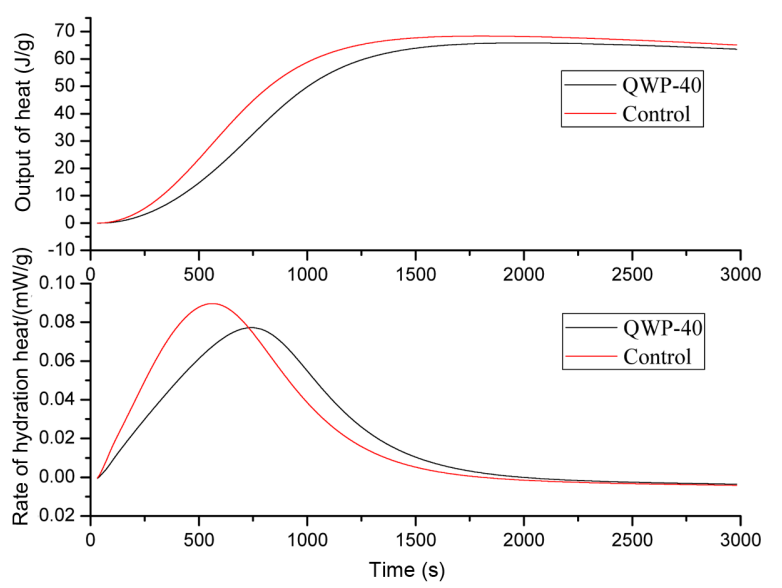

Fig. 4. Curve of hydration exothermic process of FGD gypsum plaster.

tive stage of crystallization, in which crystal nuclei had yet to grow up and overlap each other. The added retarder suppressed nucleation and extended the readiness time of crystallization. Therefore, the peak of the hydration-heat 
release rate decreased, and the peak appeared later. The plaster started to crystallize sharply, and crystals overlapped a lot to form a crystalline structure net after the final setting, so the rate of hydration-heat release increased rapidly. Compared with the control sample, QWP-40 gave rise to a significant decrease in the early hydration heat quantity. This indicates that QWP-40 has a clear inhibitory effect on the early hydration process of building gypsum.

\subsection{Ion concentration in liquid phase and degree of supersaturation}

The ion concentration in liquid phase and the degree of supersaturation had a profound impact on the crystal nucleus formation and the crystal growth of dihydrate gypsum. Researching the ion concentration in liquid phase and the change of supersaturation degree could be conducive to understanding the influence on the nature of the retarder and the hydration process of building gypsum. In this experiment, the proportion of retarder was $0.2 \%$ and the watergypsum ratio was $10: 1$. The solution was filtered and extracted, and then the $\mathrm{Ca}^{2+}$ and $\mathrm{SO}_{4}^{2-}$ concentrations in the hydrated gypsum were measured within a certain period of time. The degree of supersaturation of dihydrate gypsum: supersaturation $=\sqrt{\left[\mathrm{Ca}^{2+}\right]\left[\mathrm{SO}_{4}^{2}\right] / \mathrm{CaSO}_{4}}$. The final results are shown in Figs. 5-7.

The solubility of semi-hydrated gypsum $\left(8.2 \mathrm{~g} / \mathrm{L}, 20^{\circ} \mathrm{C}\right)$ is much higher than that of dihydrate gypsum $\left(2.08 \mathrm{~g} / \mathrm{L}, 20^{\circ} \mathrm{C}\right)$. Thus, semi-hydrated gypsum quickly forms a highly supersaturated solution after contact with water. The crystallization of dihydrate gypsum not only removes $\mathrm{Ca}^{2+}$ and $\mathrm{SO}_{4}^{2-}$ concentration, but also sharply reduces the degree of supersaturation. When the retarder was added, the ion concentration in the initial liquid phase and the saturation of the gypsum was slightly lower than the control sample, but the rate of the hydration reaction process slowed significantly. This indicates that QWP-40 had little effect on the dissolution course of the building gypsum; it mainly delayed the time of crystallization and the crystal growth process.

\subsection{XRD analysis}

In Fig. 8, curve a is the diffraction pattern of building gypsum with $0.2 \%$ QWP - 40, and curve $\mathrm{b}$ is the diffraction pattern without retarder. Curve a shows that the major phase was dihydrate gypsum, but the diffraction intensity decreased obviously because of the decrease of intact structured crystallization and deterioration of the crystalline structure of the dihydrate gypsum.

\subsection{Impact on the morphology of dihydrate gypsum crystals}

Figure 9 shows SEM photos displaying the morphology of dihydrate gypsum crystals. Fig. 9(a) shows the typical gypsum crystal morphology with $1000 \times$ magnification, in which the needle crystal plates with large aspect ratios (length/ diameter) mainly overlap with each other numerously. Fig. 9(b) shows the morphology of gypsum crystals with $1000 \times$ mag-

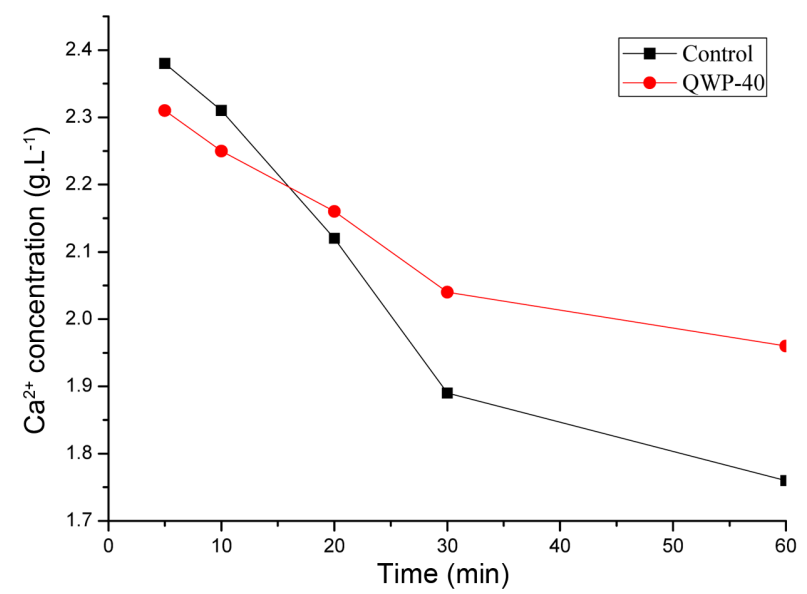

Fig. 5. Influence of retarder on the $\mathrm{Ca}^{2+}$ concentration in liquid phase.

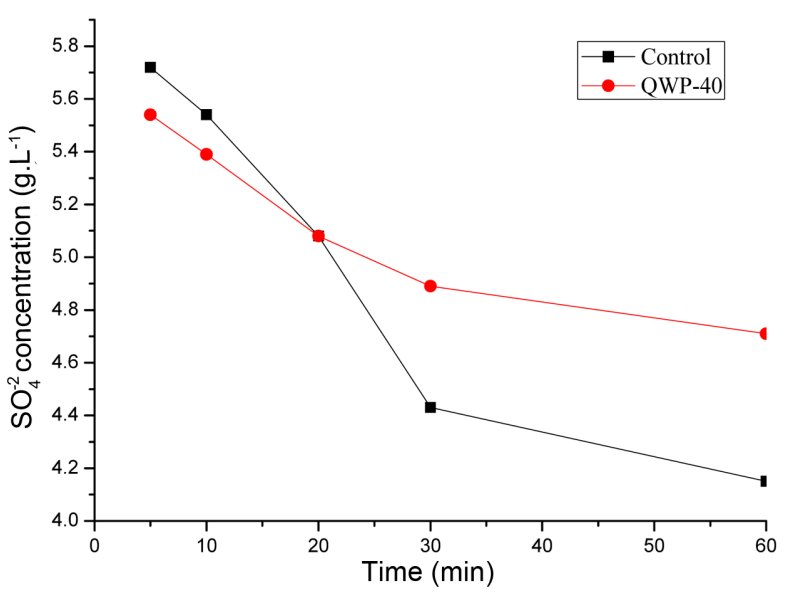

Fig. 6. Influence of retarder on the $\mathrm{SO}_{4}{ }^{2-}$ concentration in liquid phase.

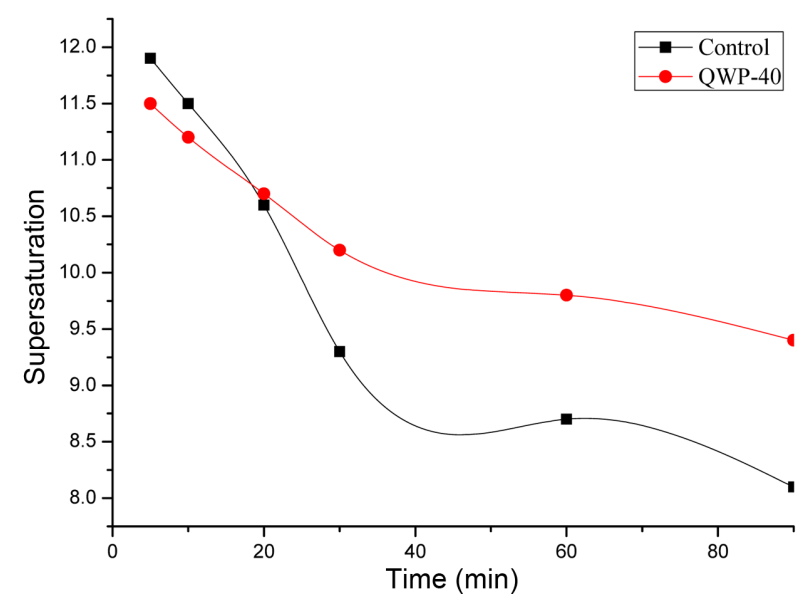

Fig. 7. Influence of plaster on the supersaturation degree with the change of time.

nification with the $0.2 \%$ retarder added. As seen in Fig. 9(b), the QWP-40 retarder may have reduced the number of needle crystal plates with a distinct and unbroken morphology. As a result, the overlapping points decreased, and the crys- 


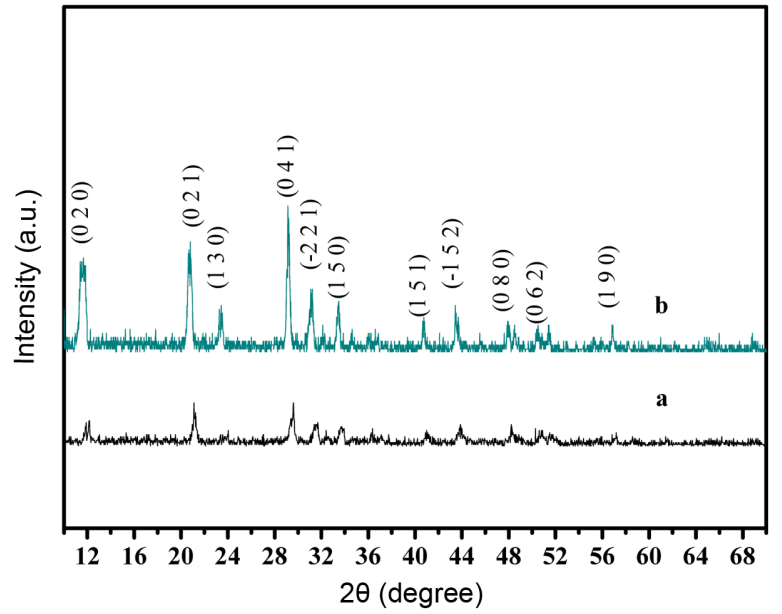

Fig. 8. XRD analysis results of building gypsum (curve a, plaster with $0.2 \%$ retarder; curve b building gypsum without retarder.).
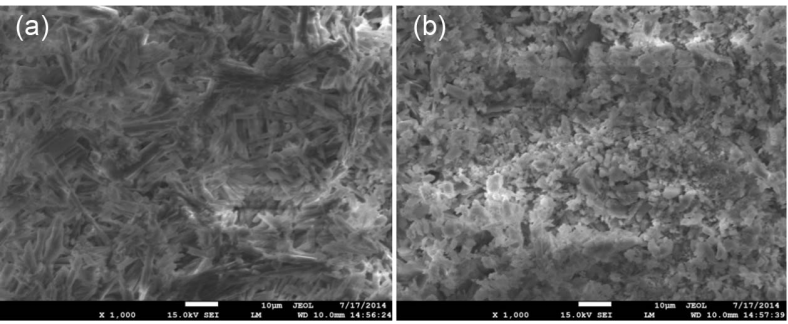

Fig. 9. SEM images of building gypsum: (a) building gypsum with $0.2 \%$ retarder and (b) building gypsum without retarder.

tal network became loose. This is clearly the cause of the slight decrease in plaster strength. As previously mentioned, needle crystal plates and crystals produce effective overlapping points that are very important in high-strength gypsum, especially the high-flexural strength gypsum. ${ }^{9}$ The results show why the strength of gypsum, especially flexural strength gypsum, decreases when retarder is added.

\subsection{Retarding mechanism}

Hydrolyzed wheat protein is a natural macromolecule polypeptide with multi-amino acids, which mainly contains amino, carboxyl, peptide bonds and other functional groups. It is soluble in water to form a colloid, and it can be combined with calcium to form a complex structure. Hydrolyzed wheat protein is produced by a chemical action with calcium, in which some active groups take the place of the weaker complexation with calcium. In this study, as seen in the element concentration on the surface of crystals, the relative content of calcium and sulfur elements on the surface of dihydrate gypsum were greatly reduced when compared with the control sample. The active groups were not only adsorbed onto the surface of the plaster by complexation, but also formed a colloid protective layer surrounding the calcium and sulfur completely.
The hydrolyzed wheat protein retarder, which covered the crystal nuclei and combined with calcium ions on the surface of the dihydrate gypsum, not only reduced the surface energy of the crystal nuclei, but also restrained the growth of the crystal nuclei. Because of the covering on the surface of crystals, their mutual contact was blocked. Thus, the formation of a crystalline network was delayed, and the hydration process was slowed down. It also can be seen from the setting time of gypsum that the retarder mainly delayed the initial setting time. Once the initial setting time was reached, the hydration process of the gypsum accelerated, and the final setting time was reached relatively quickly.

When the QWP-40 retarder was added, the number of needle crystal plates with a distinct and unbroken morphology may be reduced. As a result, the number of overlapping points decreased and the crystal network became loose; thus, the strength of plaster slightly decreased.

In the retarding mechanism of QWP-40, chemical adsorption takes place on the surface of a newly formed dihydrate gypsum crystal nucleus, and then the hydrolyzed wheat protein retarder covers the crystal nucleus and combines with calcium ions on the surface of the dihydrate gypsum, thereby reducing the surface energy of the crystal nucleus, restraining the growth of the crystal nucleus, and prolonging the setting time.

\section{Conclusion}

a. Hydrolyzed wheat protein retarder had a significant retarding action when mixed with building gypsum. The hydration exothermic process of building gypsum slowed down, he setting time increased, and the strength of the plaster slightly decreased.

b. QWP-40 had a significant influence on the morphology of dihydrate gypsum crystals. When the retarder was added, the number of needle crystal plates with a distinct and unbroken morphology is reduced. As a result, the number of overlapping points decrease and the crystal network becomes loose.

c. The hydrolyzed wheat protein retarder, which covers crystal nuclei by chemical adsorption and combines with calcium ions on the surface of dihydrate gypsum, reduces the surface energy of the crystal nuclei, restrains their growth, and prolongs the setting time.

\section{Acknowledgments}

This work was jointly supported by the special funds for the protection of Huizhou architecture of Anhui Province (2013YJ103), the National Science and Technology Support Project of China (2011BAJ03B04), the Natural Science Fund of Education Department of Anhui Province (KJ2012A058).

\section{REFERENCES}

1. P. Jiahui, Z. Jianxin, C. Mingfeng, W. Li, and Q. Jindong, 
"Influence of Macromolecule-Type Retarders on the Hydration Process of Building Gypsum and Its Retarding Mechanism," J. Chin. Ceram. Soc., 36 [7] 896-900 (2008).

2. A. Ersen, A. Smith, and T. Chotard, "Effect of Malic and Citric Acid on the Crystallisation of Gypsum Investigated by Coupled Acoustic Emission and Electrical Conductivity Techniques," J. Mater. Sci., 41 [21] 7210-17 (2006).

3. K. M. Song, J. Mitchell, H. Jaffel, and L. F. Gladden, "Simultaneous Monitoring of Hydration Kinetics, Microstructural Evolution, and Surface Interactions in Hydrating Gypsum Plaster in the Presence of Additives," $J$. Mater. Sci., 45 [19] 5282-90 (2010).

4. G. Leimen, "Influence of Various Retarders on the Crystallzation and Strength of Plaster of Paris," Zement-Kalk-Gips, 42 229-32 (1989).

5. C. Solberg and S. Hansen, "Dissolution of $\mathrm{CaSO}_{4} \cdot 1 / 2 \mathrm{H}_{2} \mathrm{O}$ and Precipitation of $\mathrm{CaSO}_{4} \cdot 2 \mathrm{H}_{2} \mathrm{O}$ A Kinetic Study by
Synchrotron X-ray Powder Diffraction,” Cem. Concr. Res., 31 [4] 641-46 (2001).

6. V. Nilles and J. Plank, "Study of the Retarding Mechanism of Linear Sodium Polyphosphates on a-calcium Sulfate Hemihydrates," Cem. Concr. Res., 42 [5] 736-44 ( 2012).

7. R. X. Magallanes-Rivera, J. I. Escalante-García, and A. Gorokhovsky, "Hydration Reactions and Microstructural Characteristics of Hemihydrate with Citric and Malic Acid," Constr. Build. Mater., 23 [3] 1298-305 (2009).

8. M. Lanzón and P. A. García-Ruiz, "Effect of Citric Acid on Setting Inhibition and Mechanical Properties of Gypsum Building Plasters," Constr. Build. Mater., 28 [1] 506-11 (2012).

9. F. Raupp-Pereira, L. Silva, A. M. Segadães, D. Hotza, and J. A. Labrincha, "Potable Water Filtration Sludge: Use as Set Retarder in One-Coat Plastering Mortars," Constr. Build. Mater., 21 [3] 646-53 (2007). 